\title{
Exact Solutions of Open Bosonic String Field Theory
}

\author{
J. Klusoň * \\ Institutionen för teoretisk fysik \\ BOX 803, SE- 75108 Uppsala, Sweden \\ E-mail: josef.kluson@teorfys.uu.se
}

\begin{abstract}
In this paper we present two solutions of open bosonic string field theory defined on D0-brane background that correspond to the change of the D0-brane position in the transverse space.
\end{abstract}

KEYWORDS: String field theory.

\footnotetext{
${ }^{*}$ On leave from Masaryk University, Brno
} 


\section{Contents}

1. Introduction 1

2. Solutions of open bosonic string field theory 2

3. Translation of D0-brane 5

4. Finite translation 10

5. Solution corresponding to the rotation in the transverse space 11

6. Conclusion 14

7. Appendix 14

\section{Introduction}

During last few years many different solutions have been studied in string field theory [1]. These solutions include tachyon vacuum and lump solutions (For review and extensive list of references, see [2, 3, 4].) and solutions representing marginal deformations [5, 6]. Other remarkable solutions have been studied recently in papers $[13,14]^{2}$. And recently there were also many papers published regarding to the string field theory formulated around closed string vacuum (See, for example [21, 22, 23, 24, 25, 26, 27, 28, 29, 30, 31, 32, 33, 34].)

In our recent paper [18] we have suggested possible exact solutions of the Berkovits superstring field theory [7, 8, 10]. Then we have extended our approach to the open bosonic string field theory [1] as well. In this paper we will continue our study. We present two solutions that correspond to the marginal deformation of the theory describing single D0-brane. The first solution corresponds to the translation in the target space orthogonal to D0-brane. Since we presume that our D0-brane lives in flat space-time its position should be irrelevant for its description. On the other hand, since D0-brane can be regarded as a defect that breaks Poincare invariance, we will see that there exist nontrivial solution. Our strategy is as follows. We use the fact that closed string theory is invariant under Poincare transformations which

\footnotetext{
${ }^{2}$ As we will see the solution given in [13] has a lot of common with the solution given in this paper.
} 
are broken by boundary conditions in the open string case describing D0-brane). Then we can construct generator of the translation that does not commute with the open string Hamiltonian thanks to Dirichlet boundary conditions, but can serve as a generator of translation that shifts position of D0-brane. We will see that this is really this case. Following our general discussion given in 18 and reviewed in section (2) we will construct solution of the open string field theory corresponding to this marginal deformation. We will see that in case of small marginal parameter the BRST operator changes exactly in the same way as we can expect from CFT analysis with accord with the very nice analysis performed in [35]. Then we will discuss possible form of the string field redefinition and we will show that after this redefinition fluctuation fields are the same as the original one as a consequence of the invariance of our configuration under translation.

Then we will extend our solution to the case of finite marginal deformation. We will see that in this case the BRST operator obtains additional term which is not expected from the CFT analysis [36]. At present we do not understand why such a term emerges. However we can still argue that thanks to the form of the new BRST operator we can perform such a field redefinition that maps the new BRST operator to the original one and string field describing the fluctuation around new solution has the same form as the original one thanks to the translation invariance of this configuration.

As the second example we present rotation of the D0-brane in the target space. As in previous case, Dirichlet boundary conditions break this rotation invariance hence we can expect that the generator of rotation does not commute with the BRST operator and hence we can get nontrivial solution. We will also discuss the problem of the field redefinition and we will argue that in this case the field changes since generator of rotation acts non trivially on tensor indexes of various string states, however thanks to the invariance of the bulk part of the BRST operator under rotation we will argue that the physical spectrum is the same. Again this can be interpreted as a trivial consequence of the isotropy of the target space.

In conclusion we outline our results and suggest possible extension of our research.

\section{Solutions of open bosonic string field theory}

As we claimed in the introduction we restrict in this paper to the case of the bosonic open string field theory [1] since generalization to the Berkovits' superstring field theory [7, 8, 10] is straightforward. This section mainly reviews results given in our previous paper [18]. 
The string field theory (SFT) action has a form ${ }^{3}$

$$
S=\frac{1}{2} \int \Phi \star Q(\Phi)+\frac{1}{3} \int \Phi \star \Phi \star \Phi
$$

Variation of the action leads to the equation of motion

$$
Q(\Phi)+\Phi \star \Phi=0
$$

According to the standard procedure let us consider the expansion of the string field around any solution of the equation of motion (2.2) $\Phi_{0}$

$$
\Phi=\Phi_{0}+\Psi
$$

After inserting (2.3) into (2.1) and using (2.2) we obtain SFT action for $\Psi$ that has the same form as the original one (2.1)

$$
S=\frac{1}{2} \int \Psi \star \tilde{Q}(\Psi)+\frac{1}{3} \int \Psi \star \Psi \star \Psi
$$

however with the modified BRST operator

$$
\tilde{Q}(X)=Q(X)+\Phi_{0} \star X-(-1)^{|X|} X \star \Phi_{0},
$$

where $X$ is any string field of ghost number $|X|$. Let us now presume that the new BRST operator $\tilde{Q}$ can be written as 18

$$
\tilde{Q}(X)=e^{-K}\left(Q\left(e^{K}(X)\right)\right)
$$

where $K$ is an operator of ghost charge equal to zero that obeys following rules [20, 26]

$$
\begin{array}{r}
K(X \star Y)=K(X) \star Y+X \star K(Y), \\
\int K(X) \star Y=-\int X \star K(Y) .
\end{array}
$$

In order to have a well defined string field theory for fluctuation field $\Psi, \tilde{Q}$ must obey following axioms [1]

$$
\begin{array}{r}
\tilde{Q}^{2}=0, \\
\tilde{Q}(X \star Y)=\tilde{Q}(X) \star Y+(-1)^{|X|} X \star \tilde{Q}(Y), \forall X, Y, \\
\int \tilde{Q}(X)=0, \forall X,
\end{array}
$$

\footnotetext{
${ }^{3}$ For very nice recent reviews of string field theory, see [2, 3, 4].
} 
where $X, Y$ are arbitrary string fields of ghost numbers $|X|$ and $|Y|$ respectively. Firstly, we can easily show that

$$
\tilde{Q}^{2}=e^{-K} Q e^{K} e^{-K} Q e^{K}=e^{-K} Q^{2} e^{K}=0
$$

since the original BRST operator $Q$ obeys (2.8) by definition. We also have

$$
\begin{array}{r}
\tilde{Q}(X \star Y)=e^{-K}\left[Q\left(e^{K}(X \star Y)\right)\right]=e^{-K}\left[Q\left(e^{K}(X) \star e^{K}(Y)\right)\right]= \\
=e^{-K}\left[Q\left(e^{K}(X)\right) \star e^{K}(Y)+(-1)^{|X|} e^{K}(X) \star Q\left(e^{K}(Y)\right)\right]= \\
=e^{-K}\left[Q\left(e^{K}(X)\right)\right] \star e^{-K} e^{K}(Y)+(-1)^{|X|} e^{-K} e^{K}(X) e^{-K}\left[Q\left(e^{K}(Y)\right)\right]= \\
=\tilde{Q}(X) \star Y+(-1)^{|X|} X \star \tilde{Q}(Y),
\end{array}
$$

where we have used

$$
e^{K}(X \star Y)=e^{K}(X) \star e^{K}(Y), e^{-K}(X \star Y)=e^{-K}(X) \star e^{-K}(Y)
$$

which follows from (2.7). We can also easily see that (2.6) obeys the third axiom in (2.8)

$$
\int \tilde{Q}(X)=\int e^{-K}\left[Q\left(e^{K}(X)\right)\right] \star \mathcal{I}=\int Q\left(e^{K}(X)\right) \star e^{K}(\mathcal{I})=\int Q\left(e^{K}(X)\right)=0
$$

where $\mathcal{I}$ is "identity", the ghost number zero field (For recent discussion of some properties of this field, see [9, 16].) which is defined as

$$
\mathcal{I} \star X=X \star \mathcal{I}=X, \forall X
$$

Then we have

$$
K(X)=K(\mathcal{I} \star X)=K(\mathcal{I}) \star X+\mathcal{I} \star K(X)=K(X) \Rightarrow K(\mathcal{I})=0
$$

and consequently

$$
e^{-K}(\mathcal{I})=e^{K}(\mathcal{I})=\mathcal{I}
$$

We have shown that the new BRST operator $\tilde{Q}$ given in (2.6) obeys all axioms (2.8). Now we must find such a solution of the equation of motion (2.2) that leads to this proposed form of the BRST operator (2.6). It is easy to see that the field [18

$$
\Phi_{0}=e^{-K_{L}(\mathcal{I})} \star Q\left(e^{K_{L}(\mathcal{I})}\right)
$$

is desired solution of the equation of motion (2.2) since we have

$$
Q\left(\Phi_{0}\right)=Q\left(e^{-K_{L}(\mathcal{I})} \star Q\left(e^{K_{L}(\mathcal{I})}\right)\right)=-e^{-K_{L}(\mathcal{I})} \star Q\left(e^{K_{L}(\mathcal{I})}\right) \star e^{-K_{L}(\mathcal{I})} \star Q\left(e^{K_{L}(\mathcal{I})}\right)
$$


and

$$
\Phi_{0} \star \Phi_{0}=e^{-K_{L}(\mathcal{I})} \star Q\left(e^{K_{L}(\mathcal{I})}\right) \star e^{-K_{L}(\mathcal{I})} \star Q\left(e^{K_{L}(\mathcal{I})}\right) .
$$

with $K$ obeying (2.7) and where $K$ is split in two parts according to [11 $K=$ $K_{L}+K_{R}$. It can be shown 18 that the solution (2.16) leads to the BRST operator (2.6). As was argued in [18] we can perform field redefinition

$$
\Psi=e^{-K}(\tilde{\Psi})
$$

that leads to the string field theory action for fluctuation field $\tilde{\Psi}$ with the BRST operator of the same form as the original one $Q$ and the string field $\tilde{\Psi}$ now describes open string theory defined around the new background configuration (For more detailed and extensive discussion of the string redefinition in the string field theory we again recommend very nice and seminal paper [35.).

In the next two sections we will continue our study and give two examples of the solutions reviewed above.

\section{Translation of D0-brane}

In this section we will describe solution of the string field theory equation of motion corresponding to the small translation in the target space. We will see that for small value of the marginal parameter $a^{I}, I=1, \ldots, 25$ we obtain such a form of the deformed BRST operator as we will get from the perturbation of the open string Lagrangian with the marginal operator. According to the general method outlined in the previous section we will perform string field theory redefinition that maps the new BRST operator to the original one and the old string field to the new string field describing fluctuation around new configuration. However thanks to the fact that our solution corresponds to the translation of a single D0-brane in the target space, it is clear that the Hilbert spaces of the original and final configuration must be isomorphic. We will confirm explicitly this conclusion very easily using the fact that the generator of the translation in the target space acts trivially on the string state describing fluctuation of D0-brane since these states carry zero momentum in the transverse direction. Then in the next section we will construct solution corresponding to the finite translation where we will see some subtle facts that are not completely clear to us at present.

For the beginning we will review well known facts about conserved charges in the bosonic string theory, following [19]. Let us consider an action for the free string [19]

$$
S=-\frac{1}{4 \pi \alpha^{\prime}} \int d^{2} \sigma \eta^{\alpha \beta} \partial_{\alpha} X^{\mu} \partial_{\beta} X^{\nu} \eta_{\mu \nu}
$$

where $\eta^{\alpha \beta}$ is two-dimensional Minkowski metric with signature $\eta_{\alpha \beta}=\operatorname{diag}(-1,1)$ and $\eta_{\mu \nu}$ is 26-dimensional Minkowski metric in the target space-time with signature 
$(-,+, \ldots,+)$. Let us suppose that this action describes string ending on D0-brane. In other words, on the boundary of the world-sheet we have Neumann boundary condition for $X^{0}$ and Dirichlet boundary condition for $X^{I}, I=1 \ldots, 25$, where the boundary of the world-sheet is at the points $\sigma=0, \pi$. Now it is easy to see that this boundary conditions break Poincare symmetry under translation, which from the point of view of two dimensional world-sheet theory is global symmetry that acts on the string coordinate $X^{\mu}(\sigma)$ as

$$
\delta X^{\mu}=\epsilon^{\mu} .
$$

Since D0-brane is placed in the point $X^{I}=0$ it is clear that the variation of $X^{I}$ is equal to zero $\delta X^{I}=0$ on the boundary of the world-sheet which leads to the breaking of the Poincare invariance. This seems to be natural fact since D0-brane can be regarded as a defect in the target space. As a result, we cannot define conserved generator of translation $P_{I}$ that commutes with the world-sheet Hamiltonian. On the other hand, $P_{I}$ is the symmetry generator of closed string theory and can be determined using Noether's method [19] as follows. Since the string action (3.1) is invariant under variation $\delta X^{\mu}=\epsilon^{\mu}$ we now presume that $\epsilon$ is a function of $\sigma, \tau$. Then we have

$$
\begin{gathered}
\delta S=-\frac{1}{2 \pi \alpha^{\prime}} \int d^{2} \sigma \eta^{\alpha \beta} \partial_{\alpha} X^{\mu} \partial_{\beta} \delta X^{\nu} \eta_{\mu \nu}=-\frac{1}{2 \pi \alpha^{\prime}} \int d^{2} \sigma \eta^{\alpha \beta} \partial_{\alpha} X^{\mu} \partial_{\beta} \epsilon^{\nu} \eta_{\mu \nu} \Rightarrow \\
\Rightarrow \partial_{\alpha}\left(\frac{1}{2 \pi \alpha^{\prime}} \eta^{\alpha \beta} \partial_{\beta} X^{\mu} \eta_{\mu \nu}\right)=0=\partial_{\alpha}\left(\eta^{\alpha \beta} P_{\beta \nu}\right)=0, P_{\beta \mu}=\frac{1}{2 \pi \alpha^{\prime}} \partial_{\beta} X^{\nu} \eta_{\nu \mu} .
\end{gathered}
$$

Then we have conserved quantity (In case of closed string or an open string obeying Neumann boundary conditions.)

$$
P_{\mu}=i \int_{0}^{\pi} d \sigma P_{0 \mu}=\frac{i}{2 \pi \alpha^{\prime}} \int_{0}^{\pi} d \sigma \dot{X}^{\nu} \eta_{\nu \mu}, \partial_{\tau} X=\dot{X}, \partial_{\sigma} X=X^{\prime} .
$$

Since $X^{\mu}(\sigma)$ is canonical coordinate we obtain from the action (3.1) its canonical conjugate momentum

$$
P_{0 \mu}(\sigma)=\frac{\delta L}{\delta \dot{X}^{\mu}(\sigma)}=\frac{1}{2 \pi \alpha^{\prime}} \dot{X}^{\nu}(\sigma) \eta_{\nu \mu}
$$

with the following commutation relation

$$
\left[P_{0 I}(\sigma), X^{J}\left(\sigma^{\prime}\right)\right]=-i \delta_{D}\left(\sigma, \sigma^{\prime}\right) \delta_{I}^{J},
$$

where $\delta_{D}\left(\sigma, \sigma^{\prime}\right)$ is a delta function that obeys Dirichlet boundary conditions so that can be written as ${ }^{4}$

$$
\delta\left(\sigma, \sigma^{\prime}\right)=\frac{1}{\pi} \sum_{n=-\infty}^{\infty} \sin n \sigma \sin n \sigma^{\prime} .
$$

\footnotetext{
${ }^{4}$ More information about delta function can be found in Appendix.
} 
Using (3.6) we can calculate commutator

$$
\begin{array}{r}
{\left[H, P_{I}\right]=\left[\frac{1}{4 \pi \alpha^{\prime}} \int_{0}^{\pi} d \sigma\left(\left(2 \pi \alpha^{\prime}\right)^{2} P_{0}^{2}(\sigma)+X^{\prime}(\sigma)^{2}\right), i \int_{0}^{\pi} d \sigma^{\prime} P_{0 I}\left(\sigma^{\prime}\right)\right]=} \\
=-\frac{1}{2 \pi \alpha^{\prime}} \int_{0}^{\pi} d \sigma \int_{0}^{\pi} d \sigma^{\prime} \partial_{\sigma} \delta_{D}\left(\sigma, \sigma^{\prime}\right) \delta_{I}^{K} X_{K}^{\prime}(\sigma)=\frac{1}{4 \pi \alpha^{\prime}} \int_{0}^{\pi} d \sigma\left(\delta_{N}(\sigma, \pi)-\delta_{N}(\sigma, 0)\right) X_{I}^{\prime}(\sigma),
\end{array}
$$

where we have used (7.5). We see that in the case of the string obeying Dirichlet boundary conditions the upper commutator is nonzero which is a consequence of the breaking of Poincare invariance with D0-brane background. On the other hand, open string with Neumann boundary conditions obeys $\left.X^{\prime}(\sigma)\right|_{0} ^{\pi}=0$ so that the upper commutator is equal to zero and hence there is conserved charge $P_{\mu}$.

Let us consider $K$ in the form

$$
K=a^{\mu} P_{\mu}=\frac{a i}{2 \pi \alpha^{\prime}} \int_{0}^{\pi} d \sigma \dot{X}^{1}, a^{\mu}=(0, a, 0, \ldots, 0) .
$$

It is easy to see, using contour arguments as in [2, 11] that $K$ given in (3.9) obeys all conditions (2.7) We can also prove that $K$ obeys (2.7) using the fact that its action on any string field $X$ can be defined as follows

$$
K(X)=K_{L}(\mathcal{I}) \star X-X \star K_{R}(\mathcal{I})=\mathcal{I} \star K_{R}(X)+K_{L}(X) \star \mathcal{I}=K(X)
$$

and operators of this form, as was reviewed recently in [26] and can be proved very easily, obey (2.7).

Let us start with situation when $a<<1$ so that we have

$$
\Phi_{0}=e^{-K_{L}(\mathcal{I})} \star Q\left(e^{K_{L}(\mathcal{I})}\right) \sim\left(\mathcal{I}-K_{L}(\mathcal{I})\right) \star Q\left(\mathcal{I}+K_{L}(\mathcal{I})\right)=Q\left(K_{L}(\mathcal{I})\right) .
$$

We can write (3.11) as

$$
\begin{array}{r}
\Phi_{0}=Q\left(K_{L}(\mathcal{I})\right)=Q_{L}\left(K_{L}(\mathcal{I})\right)+Q_{R}\left(K_{L}(\mathcal{I})\right)= \\
=Q_{L}\left(K_{L}(\mathcal{I})\right)+K_{L}\left(Q_{R}(\mathcal{I})\right)=Q_{L}\left(K_{L}(\mathcal{I})\right)-K_{L}\left(Q_{L}(\mathcal{I})\right)=\left[Q_{L}, K_{L}\right](\mathcal{I})
\end{array}
$$

using [11]

$$
Q_{L}(\mathcal{I})=-Q_{R}(\mathcal{I})
$$

and

$$
\begin{aligned}
& Q_{R}\left(K_{L}(\mathcal{I})\right)=\mathcal{I} \star Q_{R}\left(K_{L}(\mathcal{I})\right)=-Q_{L}(\mathcal{I}) \star K_{L}(\mathcal{I})= \\
= & Q_{L}(\mathcal{I}) \star K_{R}(\mathcal{I})=-K_{L}\left(Q_{L}(\mathcal{I})\right) \star \mathcal{I}=-K_{L}\left(Q_{L}(\mathcal{I})\right) .
\end{aligned}
$$

Let us define

$$
\Phi_{0}=D_{L}(\mathcal{I})=\left[Q_{L}, K_{L}\right](\mathcal{I})
$$


Then the new BRST operator $Q^{\prime}$ has the form

$$
Q^{\prime}(X)=Q(X)+D_{L}(\mathcal{I}) \star X-(-1)^{|X|} X \star D_{L}(\mathcal{I})=[Q-D](X)
$$

using

$$
\begin{array}{r}
D_{L}(\mathcal{I}) \star X=-\mathcal{I} \star D_{R}(X)=-D_{R}(X), \\
-(-1)^{|X|} X \star D_{L}(\mathcal{I})=(-1)^{|X|} X \star D_{R}(\mathcal{I})=-D_{L}(X), \\
D_{L}(X) \star Y=-(-1)^{X} X \star D_{R}(Y) .
\end{array}
$$

The last formula can be proven in the same way as in [11. Let us calculate the commutator $[Q, K]$. Since we have

$$
\begin{aligned}
Q= & \frac{1}{\pi} \int_{0}^{\pi} d \sigma J_{0}(\sigma)=\frac{1}{\pi} \int_{0}^{\pi} d \sigma c^{\alpha}(\sigma) T_{\alpha 0}(\sigma)+Q_{\text {ghost }}= \\
& =\frac{1}{\pi} \int_{0}^{\pi} d \sigma\left[c^{0} \frac{1}{2}\left(\dot{X}^{2}+X^{\prime 2}\right)+c^{1} \dot{X} X^{\prime}\right]+Q_{\text {qhost }}
\end{aligned}
$$

where $Q_{\text {qhost }}$ denotes the pure ghost part of the BRST operator which is not important for our calculation and $T_{0 \alpha}$ is the matter stress energy tensor. We see that we must calculate following commutators

$$
\begin{gathered}
{\left[T_{00}(\sigma), P\left(\sigma^{\prime}\right)\right]=\frac{1}{2}\left[X^{\prime 2}(\sigma), P\left(\sigma^{\prime}\right)\right]=i \partial_{\sigma} \delta_{D}\left(\sigma, \sigma^{\prime}\right) X^{\prime}(\sigma),} \\
{\left[T_{10}(\sigma), P\left(\sigma^{\prime}\right)\right]=\left[\dot{X}(\sigma) X^{\prime}(\sigma), P\left(\sigma^{\prime}\right)\right]=i \dot{X}(\sigma) \partial_{\sigma} \delta_{D}\left(\sigma, \sigma^{\prime}\right),}
\end{gathered}
$$

where we have used the notation

$$
P_{01}(\sigma)=P(\sigma), X^{1}(\sigma)=X(\sigma)
$$

Then we can write

$$
\begin{array}{r}
D=[Q, K]=\frac{a i}{\pi} \int_{0}^{\pi} d \sigma \int_{0}^{\pi} d \sigma^{\prime}\left[J_{0}(\sigma), P\left(\sigma^{\prime}\right)\right]= \\
=\frac{a i}{\pi} \int_{0}^{\pi} d \sigma \int_{0}^{\pi} d \sigma^{\prime}\left[c^{0}(\sigma)\left[T_{00}(\sigma), P\left(\sigma^{\prime}\right)\right]+c^{1}(\sigma)\left[T_{10}(\sigma), P\left(\sigma^{\prime}\right)\right]\right] \\
= \\
=\frac{a i}{\pi} \int_{0}^{\pi} d \sigma \int_{0}^{\pi} d \sigma^{\prime}\left[c^{0}(\sigma) i \partial_{\sigma} \delta_{D}\left(\sigma, \sigma^{\prime}\right) X^{\prime}(\sigma)+i c^{1}(\sigma) \partial_{\sigma} \delta_{D}\left(\sigma, \sigma^{\prime}\right) \dot{X}(\sigma)\right]= \\
=\frac{a}{\pi} \int_{0}^{\pi} d \sigma\left[c^{0}(\sigma) X^{\prime}(\sigma)\left(\delta_{N}(\sigma, \pi)-\delta(\sigma, 0)\right)+c^{1}(\sigma) \dot{X}(\sigma)\left(\delta_{N}(\sigma, \pi)-\delta_{N}(\sigma, 0)\right)\right]= \\
=\frac{a}{\pi} \int_{0}^{\pi} d \sigma\left[\left(c^{+} \partial_{+} X-c^{-} \partial_{-} X\right)\left(\delta_{N}(\sigma, \pi)-\delta_{N}(\sigma, 0)\right)\right],
\end{array}
$$


using (7.5) and also where we have used the notation

$$
\begin{array}{r}
\sigma^{+}=\tau+\sigma, \sigma^{-}=\tau-\sigma \\
\partial_{+}=\frac{\partial}{\partial \sigma^{+}}=\frac{1}{2}\left(\frac{\partial}{\partial \tau}+\frac{\partial}{\partial \sigma}\right), \partial_{-}=\frac{\partial}{\partial \sigma^{-}}=\frac{1}{2}\left(\frac{\partial}{\partial \tau}-\frac{\partial}{\partial \sigma}\right) \\
c^{+}=c^{0}+c^{1}, c^{-}=c^{0}-c^{1} \\
c^{+} \partial_{+} X-c^{-} \partial_{-} X=\frac{1}{2}\left[\left(c^{0}+c^{1}\right)\left(\dot{X}+X^{\prime}\right)-\left(c^{0}-c^{1}\right)\left(\dot{X}-X^{\prime}\right)\right]= \\
=\frac{1}{2}\left[c^{0} \dot{X}+c^{0} X^{\prime}+c^{1} \dot{X}+c^{1} X^{\prime}-c^{0} \dot{X}+c^{0} X^{\prime}+c^{1} \dot{X}-c^{1} X^{\prime}\right]=c^{0} X^{\prime}+c^{1} \dot{X} .
\end{array}
$$

From (3.16) and (3.22) we can conclude that our solution leads to the deformation of the stress energy tensor ${ }^{5}$

$$
T_{++}^{\prime}=T_{++}-\partial_{+} X\left(\delta_{N}(\sigma, \pi)-\delta_{N}(\sigma, 0)\right), T_{--}^{\prime}=T_{--}+\partial_{-} X\left(\delta_{N}(\sigma, \pi)-\delta_{N}(\sigma, 0)\right)
$$

using

$$
\begin{aligned}
T_{++}=\frac{1}{2}\left(T_{00}+T_{10}\right), T_{--} & =\frac{1}{2}\left(T_{00}-T_{10}\right) \\
c^{+} T_{++}+c^{-} T_{--} & =c^{0} T_{00}+c^{1} T_{10} .
\end{aligned}
$$

In other words, our solution corresponds to the marginal deformation of the worldsheet theory in precise the same way as in [35].

Now we can ask the question of the field redefinition. Recall, that according to our general discussion in section (2) we know that after suitable field redefinition the new BRST operator $Q^{\prime}$ is mapped to the original BRST operator $Q$ and the new string field is given as

$$
\tilde{\Psi}=e^{K}(\Psi)
$$

The operator $K$ acts trivially on all original fields $\Psi$ since all string states corresponding to the string living on D0-brane do not carry momentum in the transverse directions and hence are invariant under the translation. This is the result that we can expect since our solution should not depend on one particular position of D0brane in the transverse space. However as we will see on the solution corresponding to the rotation that open string states transform non trivially as a consequence of their nontrivial tensor structure.

Now let us turn to the problem of the finite translation using our general prescription given in the (2) section.

\footnotetext{
${ }^{5}$ Similar construction appeared in case of relevant perturbation in 17 .
} 


\section{Finite translation}

Let us consider the case of finite deformation where the solution is given as

$$
\Phi_{0}=e^{-K_{L}(\mathcal{I})} \star Q\left(e^{K_{L}(\mathcal{I})}\right) .
$$

In order to calculate $\Phi_{0}$ we define following function [12]

$$
F(t)=e^{-K_{L}(\mathcal{I}) t} \star Q\left(e^{K_{L}(\mathcal{I}) t}\right), \Phi_{0}=F(1)
$$

so that

$$
F(1)=F(0)+\sum_{n=1}^{\infty} \frac{1}{n !} \frac{d^{n} F(0)}{d^{n} t}
$$

where 12

$$
\begin{array}{r}
\frac{d^{n} F(t)}{d t^{n}}=e^{-t K_{L}(\mathcal{I})} \star\left[\left[Q\left(K_{L}(\mathcal{I})\right), K_{L}(\mathcal{I})\right], \ldots, K_{L}(\mathcal{I})\right] \star e^{K_{L}(\mathcal{I}) t}, n>1, \\
F(0)=Q(\mathcal{I})=0, \frac{d F(0)}{d t}=Q\left(K_{L}(\mathcal{I})\right)=[Q, K]_{L}(\mathcal{I}) .
\end{array}
$$

We see that we must calculate commutators given in (4.4). The first commutator was calculated in the (3) section with the result (3.22)

$$
[Q, K]=\frac{a}{\pi} \int_{0}^{\pi} d \sigma\left[\left(c^{+} \partial_{+} X-c^{-} \partial_{-} X\right)\left(\delta_{N}(\sigma, \pi)-\delta_{N}(\sigma, 0)\right)\right] .
$$

Next term is equal to

$$
\frac{d^{2} F(0)}{d^{2} t}=-K_{L}(\mathcal{I}) \star[Q, K]_{L}(\mathcal{I})+[Q, K]_{L}(\mathcal{I}) \star K_{L}(\mathcal{I})=-[[Q, K], K]_{L}(\mathcal{I})=E_{L}(\mathcal{I})
$$

and consequently we obtain (Up to the second order, we will see that commutators of higher order vanish.)

$$
\begin{array}{r}
Q^{\prime}(\Psi)=(Q-D)(\Psi)+\frac{1}{2} E_{L}(\mathcal{I}) \star \Psi-(-1)^{|\Psi|} \frac{1}{2} \Psi \star E_{L}(\mathcal{I})= \\
=(Q-D)(\Psi)-\frac{1}{2} \mathcal{I} \star E_{R}(\Psi)-\frac{1}{2} E_{L}(\Psi) \star \mathcal{I}=\left(Q-[Q, K]+\frac{1}{2}[[Q, K], K]\right)(\Psi),
\end{array}
$$

where

$$
\begin{array}{r}
{[[Q, K], K]=\left[\frac{a}{\pi} \int_{0}^{\pi} d \sigma\left\{c^{0}(\sigma) X^{\prime}(\sigma)\left(\delta_{N}(\sigma, \pi)-\delta_{N}(\sigma, 0)\right)\right\}, i a \int_{0}^{\pi} d \sigma^{\prime} P\left(\sigma^{\prime}\right)\right]=} \\
=-\frac{i a^{2}}{\pi} \int_{0}^{\pi} d \sigma \int_{0}^{\pi} d \sigma^{\prime}\left[c^{0}(\sigma) \partial_{\sigma} i \delta_{D}\left(\sigma^{\prime}, \sigma\right)\left(\delta_{N}(\sigma, \pi)-\delta_{N}(\sigma, 0)\right)\right]= \\
=-\frac{a^{2}}{\pi} \int_{0}^{\pi} d \sigma\left[c^{0}(\sigma)\left(\delta_{N}(\sigma, \pi)-\delta_{N}(\sigma, 0)\right)^{2}\right] .
\end{array}
$$


Now it is clear that commutators of higher order vanish and we obtain the final form of the shifted BRST operator

$$
\begin{aligned}
& Q^{\prime}=Q-\frac{a}{\pi} \int_{0}^{\pi} d \sigma {\left[\left(c^{+} \partial_{+} X-c^{-} \partial_{-} X\right)\left(\delta_{N}(\sigma, \pi)-\delta_{N}(\sigma, 0)\right)\right]+} \\
&+\frac{a^{2}}{4 \pi} \int_{0}^{\pi} d \sigma\left[\left(c^{+}+c^{-}\right)\left(\delta_{N}(\sigma, \pi)-\delta_{N}(\sigma, 0)\right)^{2}\right] .
\end{aligned}
$$

We see the remarkable fact that the deformation of the BRST operator is the same as in the CFT description [35, 36] in case of the matter sector, however we also see that there is an additional term which is in fact singular on the boundary and which contains the ghost fields only. At present, we do not completely understand reasons and meanings of this term, it is possible that more detailed analysis performed according to the paper [35] will explain emergence of this term. We will return to this question in future work.

However in spite of the fact that we have such a unusual additional term, we can still see that according to the general construction given in the (2) section after performing field redefinition with the operator $K$ the new BRST operator (4.9) is mapped to the original one and the original string field $\Psi$ maps as follows

$$
\tilde{\Psi}=e^{K}(\Psi)=\Psi
$$

which is again consequence of the fact that any string field does not carry momentum in the transverse dimensions and hence are invariant under the action of $e^{K}$.

In this section we gave an example of the exact string field theory solution corresponding to the translation of D0-brane in the transverse space. We have seen that this solution is in some sense very simple since the string field theory description of D0-brane should not depend on the position of D0-brane in the transverse space. On the other hand, as was argued in [5, 6, 35] problem of marginal deformation is quiet nontrivial in the context of the string field theory so that we believe that our approach could be helpful in further study of the string field theory. In the next section we give the second example corresponding to the rotation of the D0-brane position in the transverse space.

\section{Solution corresponding to the rotation in the transverse space}

Let us consider D0-brane that is localized in any point in the transverse space with coordinates $Y^{I}, I=1, \ldots, 25$ and consider solution of the string field theory equation of motion that corresponds to the rotation of this D0-brane. As in previous example given in (3) section Dirichlet boundary conditions explicitly break rotation invariance. 
However we can still expect that the theory should not depend on the position of D0brane in the transverse space. Let us consider following transformation of coordinates

$$
\delta X^{I}=\omega_{J}^{I} X^{J}, \omega_{J}^{I}=\text { const } .
$$

It is easy to see that the world-sheet Lagrangian is invariant under this transformation. Then the generator of rotation symmetry is given [19]

$$
M^{I J}=i \int_{0}^{\pi} d \sigma J^{0 I J}=\frac{i}{4 \pi \alpha^{\prime}} \int_{0}^{\pi} d \sigma\left(X^{I} \dot{X}^{J}-X^{J} \dot{X}^{I}\right) .
$$

Let us consider string field

$$
\Phi_{0}=Q\left(\omega_{I J} M_{L}^{I J}(\mathcal{I})\right)=D_{L}(\mathcal{I}) ; D_{L}=\left[Q, \omega_{I J} M^{I J}\right]_{L}
$$

It is clear that $\Phi_{0}$ solves the linearised equation of motion $Q(\Phi)=0$ thanks to the nilpotence of $Q$. As in previous case the new BRST operator can be written as

$$
\begin{array}{r}
Q^{\prime}=Q-D, D=\left[Q, \omega_{I J} M^{I J}\right]=\left[Q_{\text {bound }}, \omega_{I J} M^{I J}\right], \\
Q_{\text {bound }}=-\frac{1}{\pi} \int_{o}^{\pi} d \sigma c^{0}(\sigma) Y^{K} \eta_{K L} X^{L L}(\sigma)\left(\delta_{N}(\sigma, \pi)-\delta_{N}(\sigma, 0)\right) .
\end{array}
$$

where we have used manifest rotation invariance of the bulk part of the BRST operator. We have also included into bulk BRST operator the term proportional $Y_{I} Y^{I} c^{0}(\sigma)\left(\delta_{N}(\sigma, \pi)-\delta_{N}(\sigma, 0)\right)^{2}$ that is manifestly invariant under rotation as well.

We have to say a few words to the fact that we have introduced boundary term $Q_{\text {bound }}$. As we argued in the previous section we can perform field redefinition and transform the BRST operator $Q+Q_{\text {bound }}$ to the original one $Q$ and we have seen that the new string field is the same as the original one, which was a consequence of the fact that D0-brane physics in trivial background does not depend on D0-brane position in the transverse space. In the same way we could start with D0-brane in the position $Y^{I}=0$ and consequently it would not have a sense to speak about the solution of the string field equation of motion corresponding to the rotation of D0-brane. However we would like to illustrate on this example an application of the general method given in (2) section to generate solution of the equation of motion hence we have introduced the boundary term in the BRST operator that reflects the fact that D0-brane is placed in the position $Y^{I}$. We will perform more interesting calculation in case of $N$ D0-branes in separate paper. It is easy to perform calculation of the commutator with the result

$$
\left[Q_{\text {bound }}, \omega_{I J} M^{I J}\right]=-\frac{1}{\pi} \int_{0}^{\pi} d \sigma c^{0}(\sigma) Y^{I} \omega_{I J} X^{\prime J}(\sigma)\left(\delta_{N}(\sigma, \pi)-\delta_{N}(\sigma, 0)\right) .
$$

As a result, the new BRST operator $Q^{\prime}$ is

$$
Q^{\prime}=Q-D=Q_{b u l k}-\frac{1}{\pi} \int_{0}^{\pi} d \sigma c^{0}(\sigma) Y^{I}\left(\eta_{I J}+\omega_{I J}\right) X^{\prime J}(\sigma)\left(\delta_{N}(\sigma, \pi)-\delta_{N}(\sigma, 0)\right)
$$


We see that our solution corresponds to the rotation in the transverse space to the new Dirichlet boundary conditions

$$
Y^{\prime K}=Y^{I}\left(\delta_{I}^{K}+\omega_{I M} \eta^{M K}\right)=\left(\delta_{I}^{K}-\eta^{K M} \omega_{M I}\right) Y^{I}
$$

It is a simple exercise to generalize previous solution to the case of finite rotation. Then we obtain

$$
\Phi_{0}=e^{-K_{L}(\mathcal{I})} \star Q\left(e^{K_{L}(\mathcal{I})}\right)=-\sum_{n=1}^{\infty} \frac{1}{n !}\left[K,[K, \ldots,[K,[K, Q]], \ldots]_{L}(\mathcal{I})=D_{L}(\mathcal{I})\right.
$$

so that the new BRST operator is equal to

$$
\begin{array}{r}
Q^{\prime}=Q_{b u l k}+Q_{b o u n d}-D=Q_{b u l k}+Q_{b o u n d}^{\prime} \\
Q_{\text {bound }}^{\prime}=-\frac{1}{\pi} \int_{0}^{\pi} d \sigma c^{0}(\sigma) Y^{I} \Lambda_{I J} X^{J}(\sigma)\left[\delta_{N}(\sigma, \pi)-\delta_{N}(\sigma, 0)\right] \\
\Lambda_{I J}=\sum_{n=0}^{\infty} \frac{1}{n !}(\omega)_{I J}^{n}, \quad,(\omega)_{I J}^{2}=\omega_{I K} \eta^{K L} \omega_{L J}, \ldots
\end{array}
$$

where we have used

$$
\begin{array}{r}
{[K,[K, Q]]=\left[\frac{1}{\pi} \int_{0}^{\pi} d \sigma c^{0}(\sigma) Y^{I} \omega_{I J} X^{\prime J}(\sigma)\left(\delta_{N}(\sigma, \pi)-\delta_{N}(\sigma, 0)\right), \omega_{K L} M^{K L}\right]=} \\
=\frac{1}{\pi} \int_{0}^{\pi} d \sigma c^{0}(\sigma)\left[\delta_{N}(\sigma, \pi)-\delta_{N}(\sigma, 0)\right] Y^{I} \omega_{I J} \eta^{J L} \omega_{K L} \partial_{\sigma} \int_{0}^{\pi} d \sigma^{\prime} \delta_{D}\left(\sigma^{\prime}, \sigma\right) X^{K}\left(\sigma^{\prime}\right)= \\
=\frac{1}{\pi} \int_{0}^{\pi} d \sigma c^{0}(\sigma) Y^{I} \omega_{I J} \eta^{J L} \omega_{L K} X^{\prime K}(\sigma)\left[\delta_{N}(\sigma, \pi)-\delta_{N}(\sigma, 0)\right]
\end{array}
$$

so that

$$
D=\frac{1}{\pi} \int_{0}^{\pi} d \sigma \sum_{n=1}^{\infty} \frac{1}{n !} Y^{I}\left(\omega^{n}\right)_{I J} X^{J}(\sigma)\left[\delta_{N}(\sigma, \pi)-\delta_{N}(\sigma)\right] .
$$

We see that the new boundary term $Q_{\text {Bound }}^{\prime}$ corresponds precisely to the situation where D0-brane is placed in the points $Y^{\prime I}$. Following our general discussion in (2) section we can perform the field redefinition that maps the new BRST operator $Q^{\prime}$ to the original one $Q$ and the original field is mapped to

$$
\Psi^{\prime}=e^{K}(\Psi)
$$

In this case however the new field $\Psi^{\prime}$ will differ from the field $\Psi$ since various components can carry different tensor components and hence $K$ acts non trivially on these string states. Following standard methods [19] it is a simple task to obtain the new form of these fields. We will not repeat this calculation here. 


\section{Conclusion}

In this paper we have presented two examples of the classical solution of the open bosonic string field theory, following our general discussion given in paper [18]. We have seen that this method gives an expected result in case of small marginal deformation corresponding to translation in transverse space to the world-volume of D0-brane, however we have seen that in the case of finite translation there is additional term that contains only ghost contribution and is in fact singular on the boundary of the world-sheet. At present we do not precisely understand nature of this term. It is possible that it can be avoided by careful CFT calculation, following [35]. We are going to make such a calculatin. On the other hand, we have argued that with the appropriate string field redefinition we can map the new BRST operator to the original one and hence we can easily avoid the problems with this term.

To sum up, we hope that our approach gives a new view on the problem of the marginal deformation in the open string field theory. We also believe that in more interesting examples, such as a marginal deformation of the configuration of $N$ D0branes, our approach could be very useful. We return to this problem in our future work.

Of course, our approach is far more complete. As we claimed above, it would be nice to prove detailed analysis based on conformal field theory techniques, following seminal paper [35]. Again we hope to return to this problem in our future research.

Acknowledgment It is my great pleasure to thank to all people at the Department of Theoretical Physics in Uppsala, especially to Joseph Minahan, Ulf Danielsson and Konstantin Zarembo for useful discussions. I am also very graceful to Ulf Lindstrom for his support of my research. This work is partly supported by EU contract HPRN-CT-2000-00122. This work is also supported by the Czech Ministry of Education under Contract No. 143100006.

\section{Appendix}

In this appendix we will discuss some properties of the delta function ${ }^{6}$

$$
\delta\left(\sigma, \sigma^{\prime}\right)_{D}=\frac{1}{\pi} \sum_{n=-\infty}^{\infty} \sin n \sigma \sin n \sigma^{\prime} .
$$

that obeys Dirichlet boundary conditions

$$
\delta(\sigma, \pi)_{D}=\frac{1}{\pi} \sum_{n=-\infty}^{\infty} \sin n \sigma \sin n \pi=0, \delta(\sigma, 0)_{D}=0
$$

\footnotetext{
${ }^{6}$ For very nice discussion, see paper [13].
} 
It is easy to see that for any function obeying Dirichlet boundary condition

$$
f(\sigma)=\sum_{m=-\infty}^{\infty} f_{m} \sin m \sigma
$$

we get

$$
\begin{array}{r}
\int_{0}^{\pi} d \sigma f(\sigma) \delta_{D}\left(\sigma, \sigma^{\prime}\right)=\frac{1}{\pi} \sum_{m, n} \int_{0}^{\pi} f_{m} \sin m \sigma \sin n \sigma \sin n \sigma^{\prime}= \\
=\frac{1}{\pi} \sum_{m, n} \int_{0}^{\pi} d \sigma \frac{1}{2}[\cos (m-n) \sigma-\cos (m+n) \sigma] \sin n \sigma^{\prime}=\sum_{m=-\infty}^{\infty} f_{m} \sin m \sigma^{\prime}=f\left(\sigma^{\prime}\right) .
\end{array}
$$

For our purposes it is also very important following formula

$$
\begin{array}{r}
\int_{0}^{\pi} d \sigma^{\prime} \partial_{\sigma} \delta\left(\sigma, \sigma^{\prime}\right)_{D}=\int_{0}^{\pi} d \sigma^{\prime} \sum_{n} \frac{n}{\pi} \cos n \sigma \sin n \sigma^{\prime}= \\
=-\sum_{n} \frac{n}{\pi} \cos n \sigma \frac{1}{n}(\cos (n \pi)-\cos (n 0))=-\left(\delta_{N}(\sigma, \pi)-\delta_{N}(\sigma, 0)\right),
\end{array}
$$

where the delta function obeying Neumann boundary conditions is defined as

$$
\delta\left(\sigma, \sigma^{\prime}\right)=\frac{1}{\pi}+\frac{1}{\pi} \sum_{n=-\infty, n \neq 0}^{\infty} \cos n \sigma \cos n \sigma^{\prime} .
$$

\section{References}

[1] E. Witten, "Noncommutative Geometry and String Field Theory," Nucl. Phys. B 268 (1986) 253.

[2] K. Ohmori, "A Review on Tachyon Condensation in Open String Field Theories," hep-th/0102085.

[3] P. J. De Smet, "Tachyon Condensation: Calculation in String Field Theory," hep-th/0109182.

[4] I. Ya. Arefeva, D. M. Belov, A. A. Giryavets, A. S. Koshelev and P. B. Medvedev, "Noncommutative Field Theories and (Super)String Field Theories," hep-th/0111208.

[5] A. Sen and B. Zwiebach, "Large marginal deformations in string field theory," J. High Energy Phys. 0010 (009) 2000, hep-th/0007153.

[6] D. Gaiotto, L. Rastelli, A. Sen and B. Zwiebach, "Patterns in Open String Field Theory Solutions," hep-th/0201159. 
[7] N. Berkovits, "Super-Poincare Invariant Superstring Field Theory," Nucl. Phys. B 459 (1996) 339, hep-th/9503099.

[8] N. Berkovits, "A New Approach to Superstring Field Theory," hep-th/9912121.

[9] I. Ellwood, B. Feng, Y. He and N. Moeller, "The Identity String Field and the Tachyon Vacuum, hep-th/0105024.

[10] N. Berkovits, "Review of open superstring field theory," hep-th/0105230.

[11] G. T. Horowitz, J. Lykken, R. Rohm and A. Strominger, "Purely Cubic Action for String Field Theory," Phys. Rev. Lett. 57 (1986) 283.

[12] J. Klusoň, "Proposal for Background Independent Berkovits' Superstring Field Theory," hep-th/0106107.

[13] T. Takahashi and S. Tanimoto, "Wilson Lines and Classical Solutions in Cubic Open String Field Theory," Prog. Theor. Phys. '06 (2001) 863, hep-th/0107046.

[14] T. Takahashi and S. Tanimoto, "Dilaton Condensation in Cubic Open String Field Theory," hep-th/0112124.

[15] D. Gaiotto, L. Rastelli, A. Sen and B. Zwiebach, "Ghost Structure and Closed Strings in Vacuum String Field Theory," hep-th/0111129.

[16] I. Kishimoto and K. Ohmori, "CFT Description of Identity String Field: Toward Derivation of the VSFT Action," hep-th/0112169.

[17] T. Z. Husain and M. Zabzine, "Bosonic Open Strings in a Tachyonic Background Field," hep-th/0005202.

[18] J. Klusoň, "Some Solutions of Berkovits' Superstring Field Theory," hep-th/0201054.

[19] Schwarz, Greene and Witten, "String theory,", Vol. 1, Cambridge University Press, 1987.

[20] L. Rastelli, A. Sen, B. Zwiebach, "String field theory around the tachyon vacuum," hep-th/0012251.

[21] L. Rastelli, A. Sen, B. Zwiebach, "Classical Solutions in String Field Theory Around the Tachyon Vacuum", hep-th/0102112.

[22] L. Rastelli, A. Sen and B. Zwiebach, "Half-strings, projectors, and multiple D-branes in vacuum string field theory,"J. High Energy Phys. 0111 (2001) 035 hep-th/0105058.

[23] D.J. Gross and W. Taylor, "Split string field theory. I,", J. High Energy Phys. 0108 (009) 2001, hep-th/0105059.

[24] D.J. Gross and W. Taylor, "Split string field theory. II,"J. High Energy Phys. 0108 (010) 2001, hep-th/0106036. 
[25] L. Rastelli, A. Sen and B. Zwiebach, "Boundary CFT construction of D-branes in vacuum string field theory,", J. High Energy Phys. 0111 (2001) 045, hep-th/0105168.

[26] L. Rastelli, A. Sen and B. Zwiebach, "Vacuum string field theory," hep-th/0106010.

[27] H. Hata and T. Kawano, "Open string states around a classical solution in vacuum string field theory,", J. High Energy Phys. 0111 (2001) 038, hep-th/0108150.

[28] G. Moore and W. Taylor, "The singular geometry of the sliver,", hep-th/0111069.

[29] A. Hashimoto and N. Itzhaki, "Observables of String Field Theory,", hep-th/0111092.

[30] D. Gaiotto, L. Rastelli, A. Sen and B. Zwiebach, "Ghost Structure and Closed Strings in Vacuum String Field Theory," hep-th/0111129.

[31] M. Schnabl, "Wedge states in string field theory," hep-th/0201095.

[32] I.Ya. Aref'eva, A.A. Giryavets and P.B. Medvedev, "NS Matter Sliver," hep-th/0112214.

[33] M. Marino, R. Schiappa, "Towards Vacuum Superstring Field Theory: The Supersliver", hep-th/0112231.

[34] I.Ya. Aref'eva, D. M. Belov and A.A. Giryavets, "Construction of the Vacuum String Field Theory on a non-BPS Brane," hep-th/0201197.

[35] A. Sen, "On the background independence of string field theory," Nucl. Phys. B 345 (1990) 551.

[36] A. Recknagel and V. Schomerus, "Moduli Spaces of D-Branes," Nucl. Phys. B 545 (1999) 233. 